\title{
A Stochastic Approach to Improve Macula Detection in Retinal Images
}

\author{
Bálint Antal and András Hajdu*
}

\begin{abstract}
In this paper, we present an approach to improve detectors used in medical image processing by fine-tuning their parameters for a certain dataset. The proposed algorithm uses a stochastic search algorithm to deal with large search spaces. We investigate the effectiveness of this approach by evaluating it on an actual clinical application. Namely, we present promising results with outperforming four state-of-the-art algorithms used for the detection of the center of the sharp vision (macula) in digital fundus images.
\end{abstract}

Keywords: biomedical image processing, simulated annealing, learning and adaptive systems

\section{Introduction}

Diabetic Retinopathy (DR) is the most common cause of blindness in the developed countries. Nowadays, the automatic screening of DR received much attention in the medical imaging community [1], [7], [9], since replacing a resource-demanding and expensive manual screening is a very challenging task. Automatic screening is based on the analysis of retinal images taken at eye hospitals. One class of the difficulties originates from the use of different kinds of retinal images, which leads to varying performance in the anatomy or lesion detection processes. Some detectors are based on machine learning, while others consider non-training approaches.

In this paper, we present a technique to improve a detection algorithm on retinal images via a learning-based approach. The idea behind this technique is to finetune the parameter setup for a certain detector. Since the selection of the optimal parameter setup usually traverses a large search space, we decided to use a stochastic approach, simulated annealing for this task. To demonstrate the effectiveness of this technique, we present a novel macula detector and show that the proposed framework improves detection performance. The contribution in this particular area is justified by the fact that the detection of macula involves the lowest number of reported works in the field of DR screening research [16]. A comparative analysis

*University of Debrecen, Hungary. E-mail: \{antal.balint, hajdu.andras\}@inf.unideb.hu 
reveals that our tuned algorithm outperforms other state-of-the-art algorithms in the field.

The main contributions of the paper are organized into the following sections:

1. A stochastic approach to improve detector performance is introduced. We also discuss the advantages of using simulated annealing over stochastic hill climbing (Section 2).

2. We show how to adopt the simulated annealing based search method to improve the performance of the proposed macula detector (Section 3).

3. A novel macula detector is proposed, which, in addition to its good performance, can be easily fine-tuned by a search algorithm (Section 3.1).

4. We define an error measure to efficiently characterize macula detection performance (Section 3.3).

5. We evaluate the performance of our macula detector using the proposed tuning also in comparison with four state-of-the-art algorithms (Section 4).

\section{A stochastic approach to improve detector per- formance}

In this section, we present our approach to select an optimal parameter setup for a detector algorithm. For this task, we have to prepare for a large search space, since these algorithms may operate with several parameters. In literature, stochastic hill climbing is often recommended [10] [13]. Stochastic hill climbing is based on the idea that using random jumps between the neighbouring elements of the search space converges faster to the extrema than using exhaustive enumerations. An element is accepted, if it provides better result than the current extremum. This approach is an effective solution for many problems, but it can get stuck in a local extrema in search spaces with many peaks.

To overcome this difficulty, we used a simulated annealing-based method. Simulated annealing [5] avoids getting stuck in local extrema by using a random acceptance function for rejected elements. That is, if an element does not provide a better result than the current one, it is still accepted if the acceptance function allows that. See Figure 1 for a visual comparison of hill climbing and simulated annealing.

The formal description of the algorithm can be given as follows:

Algorithm 1.: Parameter setup selection by simulated annealing.

\section{Input:}

- An initial temperature $T \in \mathbb{R}$.

- A minimal temperature $T_{\min } \in \mathbb{R}$. 


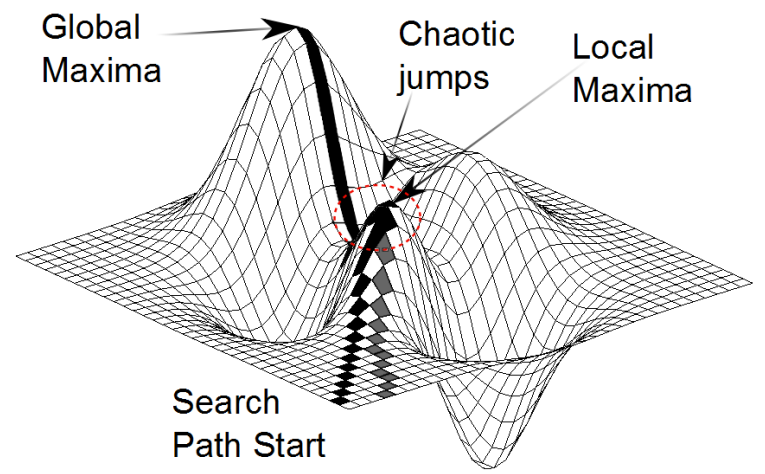

Figure 1: The path of the hill climbing and the simulated annealing algorithm is represented with gray and black colors, respectively. While hill climbing reaches only the local optimum, simulated annealing can continue towards the global optimum by using chaotic jumps.

- A temperature change $q \in \mathbb{R}$ with $(0 \leq q \leq 1)$.

- A search space $S \subset \mathbb{R}^{n}$ with $s \in S$ is a parameter setup.

- A function $r(X)$, which chooses a random element $x$ from a set $X$.

- A function accept : $\mathbb{R}^{4} \rightarrow\{$ true, false $\}$, which is defined as the follows:

$$
\operatorname{accept}\left(e, e_{i}, T, y\right)= \begin{cases}\text { true, } & \text { if } \exp \left(\frac{e-e_{i}}{T}\right)>y \\ \text { false, } & \text { otherwise }\end{cases}
$$

- An energy function $E: S \rightarrow \mathbb{R}$.

Output: $s_{\text {optimal }} \in S$, where $E\left(s_{\text {optimal }}\right)=\min _{s \in S} E(s)$. That is, $s_{\text {optimal }}$ is the parameter setup minimizing the energy function $E$.

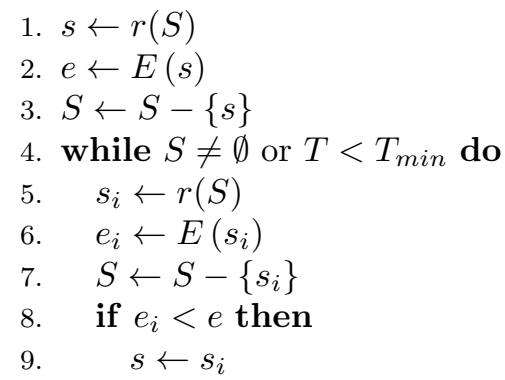




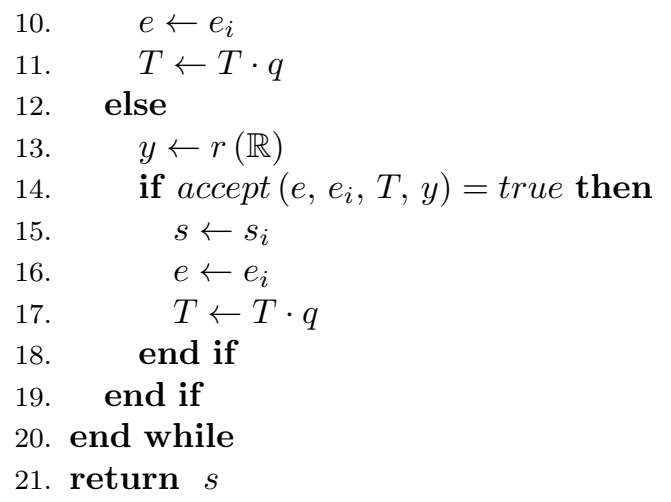

\section{Using the proposed approach: an example}

In this section, we present an example to demonstrate the power of the proposed method. For this task, we chose a novel approach for macula detection in retinal images, which algorithm requires only two parameter to be optimized. Our proposed approach for obtaining the optimal parameter setup can be adapted to any similar problem, as well.

\subsection{Macula detection}

The macula is the central region of sharp vision in the human eye, with its center referred to as the fovea (see Figure 2). Any lesions (e.g. microaneurysms) which appear here can lead to severe loss of vision. Therefore, the efficient detection of the macula is essential in an automatic screening system for diabetic retinopathy.

\subsection{A novel algorithm for macula detection}

In this section, we present a novel approach to detect macula in a retinal image. As we can see later on, this algorithm outperforms state-of-the-art macula detectors with the use of the proposed framework for optimal parameter setup.

The proposed macula detection algorithm can be formulated as follows:

Algorithm 2.: A novel macula detector

\section{Input:}

- A digital retinal image $I$ in 24 bit $R G B$ format.

- A parameter $q \in \mathbb{R}$ with $0 \leq q \leq 1$ to adjust of the mask size in the median filtering step.

- A threshold $t \in[-255, \ldots, 255]$. 


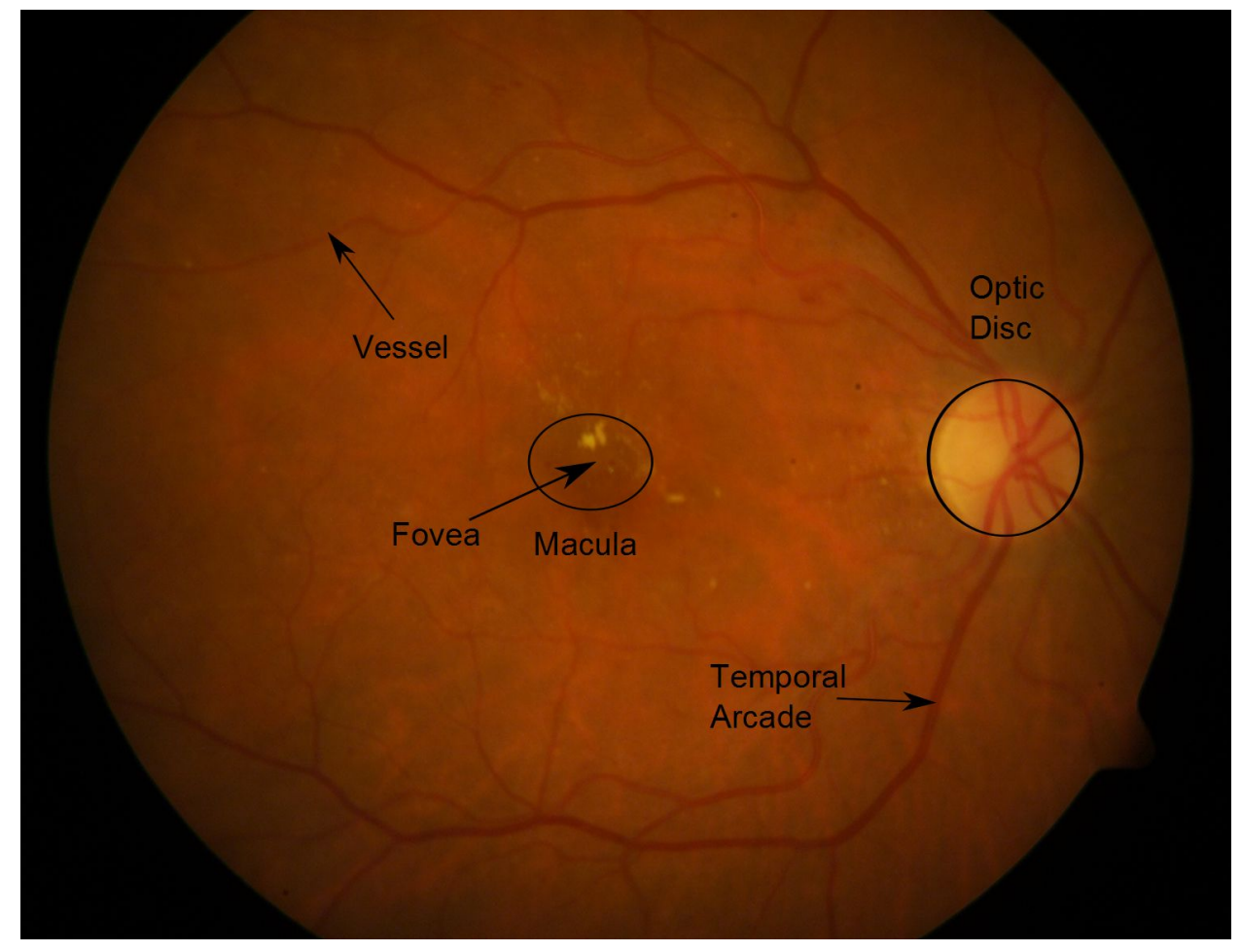

Figure 2: A sample fundus image with the main anatomical parts annotated.

Output: An image containing the macula region of the eye.

1. Extract the green intensity channel $G$ from $I$.

2. Let $A=\lceil\operatorname{Min}($ width $(I)$, height $(I)) \cdot q\rceil$.

3. Produce image $M$ with the same size as $G$ by applying median filtering [12] on $G$ with a mask size $A \times A$.

4. Create the difference image $D=G-M$.

5. Produce a binary image $B$ by assigning all pixels with larger intensity than $t$ in the $D$ to the foreground, while the rest to its background.

6. Select the largest binary component to locate the macula.

The results after each step of the algorithm can also be observed in Figure 3. 


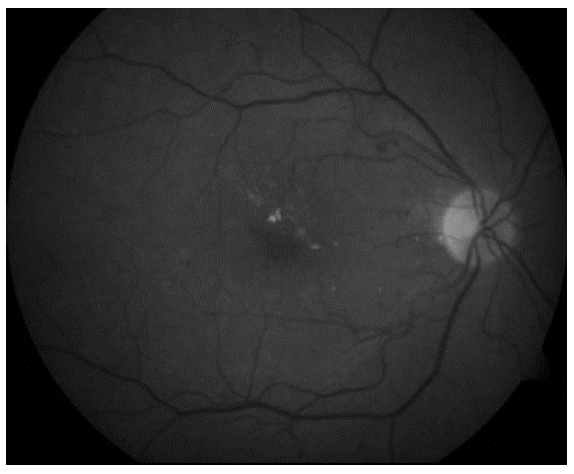

(a)

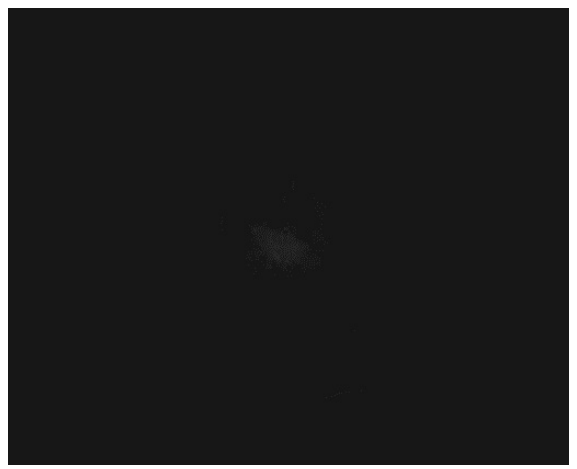

(c)

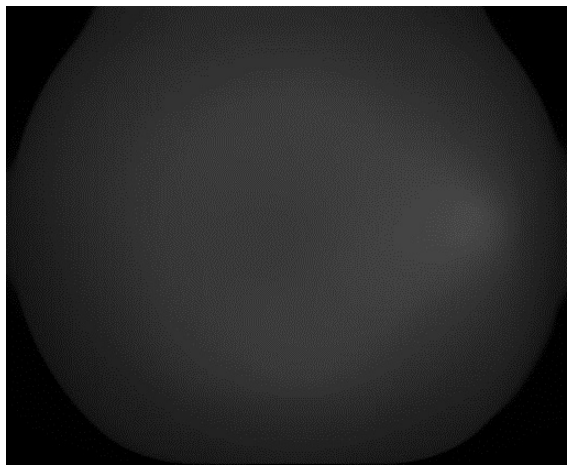

(b)

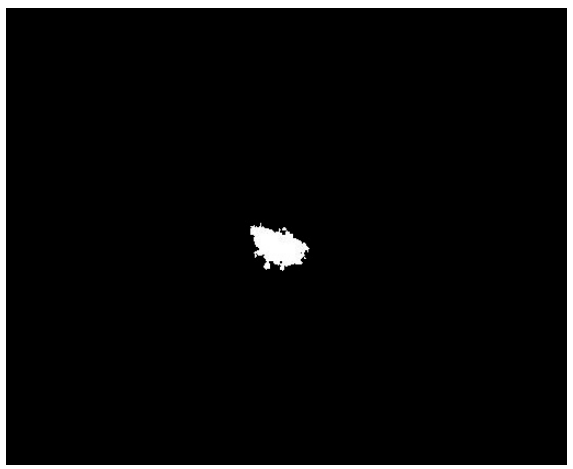

(d)

Figure 3: Steps of the proposed macula detection: (a) The green channel of the input image. (b) The result of the median filtering. (c) The difference image. (d) The binary image after thresholding and largest component selection.

\subsection{Error measurement of macula detectors}

To select the optimal parameter setup for the above detector algorithm, we need a proper energy function to be minimized. An obvious choice for this task is to minimize the distance of the centroid of the macula found by the detector and the manually selected center of the macula for each image in a dataset. To avoid overtraining, we also take into account the distance from the optic disc (see Figure 2 ), as the macula and the optic disc are spatially constrained [11].

Thus, we define the following energy function for this problem:

$$
E=\sum_{I \in D S} d\left(M_{a l g}(I), M_{h m}(I)\right)+\sum_{I \in D S}\left|d\left(M_{a l g}(I), O\right)-M O_{a v g}\right|,
$$

where 
- $D S$ is the dataset,

- $d$ denotes the 2D Euclidean distance,

- $M_{a l g}$ is the centroid pixel of the detected macula,

- $M_{h m}$ is the manually selected macula center,

- $O$ is the manually selected optic disc center,

- $M O_{a v g}$ is the average Euclidean distance of the manually selected macula and optic disc center for the dataset $D S$.

\section{Comparative results}

We evaluate our method by comparing it with four other state-of-the-art macula detectors (Section 4.1) on different datasets (Section 4.2). As our results will show, the novel macula detector outperforms the others after finding its optimal parameter setup.

\subsection{State-of-the-art macula detection algorithms}

In this section, we list four macula detection algorithms, which are involved in our comparative analysis. The parameters of the algorithms were set according to the corresponding recommendations in literature.

\subsubsection{Petsatodis et al. [8]}

In [8] a region of interest (ROI) is defined to process macula detection. A Gaussian low-pass filter is applied to smooth the image. The statistical mean and standard deviation of the ROI area are used to compute a threshold for segmentation to get binary objects. The object that is located nearest to the center of the ROI is labelled as macula. Its center of mass is considered to be the center of the macula. However, we did some modification to this approach, because it is not mentioned how this ROI is defined; therefore we applied the smoothing to the whole image using a large kernel $(70 \times 70$ pixels with $\sigma=10)$ so that vascular network and small patches do not interfere in detection. Then, an iterative thresholding process is launched to generate a set of binary images corresponding to different threshold values. In each binary image, the component satisfying the area and distance from the center constraints are identified, and the component found nearest to the center with minimum area is marked as macula.

\subsubsection{Sekhar et al. [11]}

In [11] a region of interest (ROI) for macula is defined regarding its spatial relationship to the optic disc. That is the portion of a sector subtended at the center of the optic disc by an angle of $30^{\circ}$ above and below the line between this center and 
the center of the retinal image disc. The macula is identified within this ROI by iteratively applying a threshold, and then applying morphological opening (erosion followed by dilation) on the resulting blob. The value of the threshold parameter is selected such that the area of the smoothed macula region is not more than $80 \%$ of that of the detected optic disc. The fovea is simply determined as the centroid of this blob.

\subsubsection{Fleming et al. [2]}

Fleming et al. [2] proposed to identify the macular region based on the information of the temporal arcade and OD center. First, the arcade is found by using semielliptical templates. Next, the optic disc is detected by using Hough transformation with circular templates having diameters from 0.7 to $1.25 \mathrm{OD}$ diameter (DD). Finally, the fovea was detected by finding the maximum correlation coefficient between the image and a foveal model. The search was restricted to a circular region with diameter $1.6 \mathrm{DD}$ centered on a point that is $2.4 \mathrm{DD}$ from the optic disc and on a line between the detected optic disc and the center of the semi-ellipse fitted to the temporal arcades.

\subsubsection{Zana et al. [17]}

Zana et al. [17] presented a region merging algorithm based on watershed cell decomposition and morphological operations for macula recognition. After noise removal, morphological closing followed by opening is performed to remove the small dark holes and white spots. A watershed based decomposition of the gradient image into cells is done, and the cell with darkest gray level inside the macula is selected as the first step of a merging algorithm. A complex criterion based on the gray values and of edges of the filtered image is calculated to merge the cells of the macula, while rejecting perifoveal inter-capillary zones in order to produce the contour of the macula.

\subsection{Datasets}

We have tested our approach on 199 images from three publicly available data sources: DiaretDB0 [3], DiaretDB1 [4] and DRIVE [15]. The characteristic properties of these datasets can be seen in Table 1 . We have selected the optimal parameter setup for each dataset using a separate training subset of a total of 60 images (20 images from each dataset). For each dataset, the ground truth are used only for parameter selection.

\subsection{Results}

Table 2 shows the selected optimal parameters for each dataset. The size parameter $q$ and the threshold parameter $t$ have been found by the proposed stochastic approach. Each dataset performed optimally using a different parameter setup. We have evaluated our approach in two aspects [6]: whether the detected macula 


\begin{tabular}{|c|c|c|c|c|c|}
\hline Dataset & Images & Normal & DR & FOV & Resolution \\
\hline DiaretDB0 & 130 & 20 & 110 & 50 & $1500 \times 1152$ \\
\hline DiaretDB1 & 89 & 5 & 84 & 50 & $1500 \times 1152$ \\
\hline DRIVE & 40 & 33 & 7 & 45 & $768 \times 584$ \\
\hline
\end{tabular}

Table 1: Properties of the datasets.

\begin{tabular}{|c|c|c|}
\hline Dataset & $q$ & $t$ \\
\hline DiaretDB0 & 0.6 & 0 \\
\hline DiaretDB1 & 0.6 & 5 \\
\hline Drive & 0.7 & 0 \\
\hline
\end{tabular}

Table 2: Parameters selected by the proposed algorithm for macula detection.

center falls into the 0.5DD (Optic Disc Diameter) distance of the manually selected macula center and we also measured the Euclidean distance of them (calculated on normalized images). Table 3 and 4 contain the quantitative results using these measures, respectively. We disclose the results for each macula detector evaluated in all dataset. For the more straight-forward comparison, we also calculated the simple average of these performance values. In the terms of the first measure, the use of the proposed algorithm on the novel macula detector resulted in a $85 \%$ average accuracy, while the second best method only earned $77 \%$. However, in the terms Euclidean error it is only third in the comparison, mainly because of its difficulties on the DRIVE database.

\begin{tabular}{|c|c|c|c|c|c|}
\hline Dataset & Petsatodis & Sekhar & Fleming & Zana & Proposed \\
\hline DiaretDB0 & $68 \%$ & $72 \%$ & $85 \%$ & $63 \%$ & $86 \%$ \\
\hline DiaretDB1 & $62 \%$ & $76 \%$ & $79 \%$ & $71 \%$ & $92 \%$ \\
\hline DRIVE & $66 \%$ & $76 \%$ & $53 \%$ & $82 \%$ & $68 \%$ \\
\hline Average & $66 \%$ & $74 \%$ & $77 \%$ & $69 \%$ & $85 \%$ \\
\hline
\end{tabular}

Table 3: Percentage of detected macula centers falling in the correct region.

\section{Conclusion}

In this paper, we have presented an approach to improve detection algorithms by fine-tuning their parameters. For this task, we have used a simulated annealingbased search algorithm. As our experiments have proved, this approach is capable of improving a detector that outperforms state-of-the-art algorithms in the field of macula detectors. As a future work, the selection of different preprocessing methods for the dataset can further improve the detection of the macula. In addition, both simulated annealing [14] and the proposed detector could be implemented in parallel to reduce their computational needs. 


\begin{tabular}{|c|c|c|c|c|c|}
\hline Dataset & Petsatodis & Sekhar & Fleming & Zana & Proposed \\
\hline DiaretDB0 & 26.59 & 26.85 & 37.82 & 24.11 & 24.02 \\
\hline DiaretDB1 & 26.32 & 27.45 & 35.67 & 24.77 & 25.72 \\
\hline DRIVE & 18.15 & 26.20 & 37.29 & 20.85 & 30.25 \\
\hline Average & 23.69 & 26.83 & 36.92 & 23.24 & 26.75 \\
\hline
\end{tabular}

Table 4: Average euclidean distance of the detected macula centers from the manually selected ones.

\section{Acknowledgement}

This work was supported in part by the János Bolyai grant of the Hungarian Academy of Sciences, and by the TECH08-2 project DRSCREEN - Developing a computer based image processing system for diabetic retinopathy screening of the National Office for Research and Technology of Hungary (contract no.: OM00194/2008, OM-00195/2008, OM-00196/2008). We also acknowledge the Moorefields Eye Hospital, London for their clinical support. We are thankful to Brigitta Nagy and Ignác Csősz for their technical assistance.

\section{References}

[1] Abramoff, M. D., Reinhardt, J. M., Russell, S. R., Folk, J. C., Mahajan, V. B., Niemeijer, M., and Quellec, G. Automated early detection of diabetic retinopathy. Ophthalmology, 117(6):1147-1154, 2010.

[2] Fleming, A. D., Philip, S., Goatman, K. A., Olson, J. A., and Sharp, P.F. Automated assessment of diabetic retinal image quality based on clarity and field definition. Investigative Ophthalmology and Visual Science, 47:1120-1125, 2006.

[3] Kauppi, T., Kalesnykiene, V., Kamarainen, J.K., Lensu, L., Sorri, I., Uusitalo, H., Kalviainen, H., and Pietila, J. Diaretdb0: Evaluation database and methodology for diabetic retinopathy algorithms. Technical report, Lappeenranta University of Technology, Lappeenranta, Finland, 2006.

[4] Kauppi, T., Kalesnykiene, V., Kmrinen, J.K., Lensu, L., Sorri, I., Raninen, A., Voutilainen, R., Uusitalo, H., Klviinen, H., and Pietil, J. Diaretdb1 diabetic retinopathy database and evaluation protocol. Proc. of the 11th Conf. on Medical Image Understanding and Analysis (MIUA2007), pages 61-65, 2007.

[5] Kirkpatrick, S., Gelatt, C. D., and Vecchi, M. P. Optimization by simulated annealing. Science, 220:671-680, May 13, 1983.

[6] Kovacs, L., Qureshi, R.J., Nagy, B., Harangi, B., and Hajdu, A. Graph based detection of optic disc and fovea in retinal images. In Soft Computing Applications (SOFA), 2010 4th International Workshop on, pages 143-148, 2010. 
[7] Niemeijer, M., van Ginneken, B., Cree, M.J., Mizutani, A., Quellec, G., Sanchez, C.I., Zhang, B., Hornero, R., Lamard, M., Muramatsu, C., Wu, X., Cazuguel, G., You, J., Mayo, A., Li, Q., Hatanaka, Y., Cochener, B., Roux, C., Karray, F., Garcia, M., Fujita, H., and Abramoff, M.D. Retinopathy online challenge: Automatic detection of microaneurysms in digital color fundus photographs. IEEE Transactions on Medical Imaging, 29(1):185-195, 2010.

[8] Petsatodis, T. S., Diamantis, A., and Syrcos, G. P. A complete algorithm for automatic human recognition based on retina vascular network characteristics. Era1 International Scientific Conference, Peloponnese, Greece, 16-17 September 2006.

[9] Ravishankar, S., Jain, A., and Mittal, A. Automated feature extraction for early detection of diabetic retinopathy in fundus images. In $C V P R$, pages 210-217. IEEE, 2009.

[10] Ruta, D. and Gabrys, B. Classifier selection for majority voting. Information Fusion, 6(1):63 - 81, 2005. Diversity in Multiple Classifier Systems.

[11] Sekhar, S., Al-Nuaimy, W., and Nandi, A. K. Automated localization of optic disc and fovea in retinal fundus images. 16th European Signal Processing Conference, Lausanne, Switzerland, 2008.

[12] Sinha, P. K. and Hong, Q. H. An improved median filter. IEEE Transactions on Medical Imaging, 9(3):345-346, 1990.

[13] Sivanandam, S. N. and Deepa, S. N. Introduction to Genetic Algorithms. Springer, 2008.

[14] So, O. and Özdamar, L. Parallel Simulated Annealing Algorithms in Global Optimization. Journal of Global Optimization, 19:27-50, 2001.

[15] Staal, J., Abramoff, M. D., Niemeijer, M., Viergever, M. A., and van Ginneken, B. Ridge-based vessel segmentation in color images of the retina. IEEE Transactions on Medical Imaging, 23:501-509, 2004.

[16] Winder, R.J., Morrow, P.J., McRitchie, I.N., Bailie, J.R., and Hart, P.M. Algorithms for digital image processing in diabetic retinopathy. Computerized Medical Imaging and Graphics, 33(8):608-622, 2009.

[17] Zana, F., Meunier, I., and Klein, J. C. A region merging algorithm using mathematical morphology: application to macula detection. In ISMM '98: Proceedings of the fourth international symposium on Mathematical morphology and its applications to image and signal processing, pages 423-430, Norwell, MA, USA, 1998. Kluwer Academic Publishers. 